\title{
AGB circumstellar envelopes: molecular observations
}

\author{
V. Bujarrabal \\ Observatorio Astronómico Nacional, Apartado 1143, E-28800 Alcalá de \\ Henares, Spain
}

\begin{abstract}
Due to the low excitation requirements and easy observation from the ground, molecular line observations probably constitute our main source of empirical knowledge on circumstellar envelopes (CSEs) around AGB stars. The $\mathrm{CO}$ rotational transitions, the most intense 'thermal' lines, are efficiently used to determine the total gas content and its spatial distribution in wide samples of objects. Thermal emission from other molecules is mainly useful in order to study their abundances and the chemical reactions taking place in CSEs. Maser lines are easily observed due to their high intensity and the flux distribution in very compact spatial spots and narrow profile spikes, characteristic of the exponential amplification; however the data interpretation is difficult due to the intricate pumping processes. The most important maser lines (of $\mathrm{SiO}, \mathrm{H}_{2} \mathrm{O}$ and $\mathrm{OH}$ ) arise from very different regions, which allows the study of various components of the CSEs. We will focus here on $\mathrm{SiO}$ masers.
\end{abstract}

\section{Excitation of molecular transitions in AGB circumstellar envelopes}

Molecular lines provide us with data on the total amount of gas in circumstellar envelopes (CSEs) around AGB stars, as well as on its structure, the physical conditions in them, and the abundances of more than 50 species. Thousands of stars have been observed. Such observations are eased by a) the very reliable, ground based observational techniques applied (mostly at $\mathrm{mm}$ wavelengths), and b) the low excitation present in CSEs, which allows that most of the gas is molecular, but is high enough to excite the molecular rotational transitions.

Molecular lines are useful to study the circumstellar gas except for very small, inner regions $\left(<10^{15} \mathrm{~cm}\right)$, that are not specifically probed by these lowexcitation lines, and the very outer regions $\left(\gtrsim 10^{18} \mathrm{~cm}\right)$, where molecules are photodissociated by the interstellar UV radiation field. The photodissociation radii are thought to vary between about $10^{16} \mathrm{~cm}$ and $10^{18} \mathrm{~cm}$; see Mamon et al. (1988) for the case of CO, and e.g. Lafont et al. (1982) and the review by A. Glassgold (in this volume) for other molecules. The largest radius values hold for the densest envelopes and most abundant molecules, due to shielding; for $\mathrm{CO}$, we often expect values for the photodissociation radii $R_{C O} \sim 10^{17} \mathrm{~cm}$.

The temperature of the circumstellar gas around AGB stars ranges between about $1000 \mathrm{~K}$ in the innermost region, $\sim 100 \mathrm{~K}$ at $\sim 10^{16} \mathrm{~cm}$, and about $20 \mathrm{~K}$ in outer layers $\left(\sim 10^{17} \mathrm{~cm}\right)$. Variations of about a factor 3 are expected from object to object, the less dense envelopes being predicted to be the hotter ones. From 
comparison between the different models for circumstellar thermodynamics (the predictions of which have been by themselves exhaustively checked with observations), the above figures seem relatively well established, with uncertainties also of about a factor 3 . Only in the very inner and outer regions the uncertainties can be larger, due to the difficult comparison with observations. For more details on the circumstellar thermodynamics see the seminal paper by Goldreich \& Scoville (1976), Justtanont et al. $(1994,1996)$ for the case of O-rich stars, Kwan \& Linke (1982) and Groenewegen et al. (1994, 1998) for the well known C-rich star IRC +10216 , and Kastner (1992) for several other cases.

With these temperatures, the circumstellar gas is expected to be always molecular, except for the outer regions, as we have mentioned. These low temperatures can almost only excite rotational transitions in the ground vibrational state, since the relevant species have vibrational energies $\gtrsim 2000 \mathrm{~K}$. For instance the first rotational level of $\mathrm{CO}$ is placed $5.5 \mathrm{~K}$ over the ground, but its first vibrational state is at more than $3000 \mathrm{~K}$. (In some cases, the absorption of stellar photons can yield a significant vibrational excitation, as we will see later.)

In this review we will mostly deal with rotational molecular lines in CSEs, discussing both maser and 'thermal' (i.e. non maser) lines at millimeter wavelengths. High- $J$ and vibrational transitions, observed in the IR, are discussed in reviews by $M$. Barlow and $S$. Höfner in this volume.

\section{CO 'thermal' emission}

$\mathrm{CO}$ is the most abundant molecule in CSEs (after $\mathrm{H}_{2}$ ), its lowest rotational transitions being the most intense molecular lines. They have been detected in about five hundred objects. The rare isotopic substitutions (mainly ${ }^{13} \mathrm{CO}$ ) can also be observed. The chemistry of $\mathrm{CO}$ is particularly well known. Its high abundance is due to its very high dissociation energy, and almost all the available $\mathrm{C} / \mathrm{O}$ atoms are locked in $\mathrm{CO}$, up to $R_{C O}$, see 3.1 .

The excitation of the low- $J$ transitions of this molecule is also easy to understand. The level structure is simple, consisting of a single rotational ladder with transitions at $2.6 \mathrm{~mm}(J=1-0), 1.3 \mathrm{~mm}(J=2-1), 0.9 \mathrm{~mm}(J=3-2)$, etc. Due to the anomalously low value of the Einstein coefficients (for instance, the A-coefficient of the CO $J=1-0$ transition is only $710^{-8} \mathrm{~s}^{-1}$ ), the population by collisions of the low-energy levels is very efficient.

If we assume that a $\mathrm{CO}$ transition is optically thin and the rotational excitation temperature $T_{\text {rot }}$ is much larger than the energy of the levels joined by the transition (and than the background radiation temperature), it is easy to show that the net photon production rate of this transition per unit time and volume is: $n_{p h} \sim A n_{u} \stackrel{\propto}{\sim} n_{C O} / F_{p a r} \stackrel{\propto}{\sim} n_{C O} / T_{\text {rot }}$. Where $A$ is the Einstein coefficient of the transition, $n_{u}$ is the population of its upper level, $n_{C O}$ is the total number of $\mathrm{CO}$ molecules per cubic centimeter, and $F_{p a r}$ is the partition function. Note that only the rotational temperature (i.e. an average of the excitation temperatures of the rotational transitions) appears, through the partition function, and not the excitation temperature of our transition. The moderate dependence on the inverse rotational temperature is remarkable. Since the radiative transition probabilities are low for $\mathrm{CO}, T_{\text {rot }}$ is often close to be thermalized, i.e. close to the kinetic temperature $T_{k}$. Detailed calculations show indeed that the 
overexcitation (relatively large $T_{\text {rot }}$ ) and approximate thermalization are widely satisfied in relevant cases (closer than $10^{16}-10^{17} \mathrm{~cm}$ ); however, $T_{\text {rot }}$ is often somewhat lower than $T_{k}$, since the population of the high- $J$ levels is difficult. This simple formulation is only illustrative of how the $\mathrm{CO}$ emission behaves in the case of overexcitation, but it shows that the dependence of the line intensity on the physical conditions is moderate in our case, which means that the model calculations must be robust and give reliable results.

However, when the overexcitation condition is not satisfied the line excitation becomes much more difficult to treat. In the limit, we are dealing with a differential population effect that is very elusive to accurate calculations. Such a case appears in the outer regions of the envelope, where the kinetic temperature and density are low. Since the volume of these regions can be enormous, the total emission from them should be dominant, in spite of underexcitation. Note that the excitation via the absorption of stellar IR photons by the vibrational transitions becomes important precisely in these outer regions; such a phenomenon is also difficult to treat in the models due to the non-local nature of the strong selfabsorption effects in the rovibrational components. We also note that the detection of these very extended, low-brightness shells is difficult, particularly in interferometric observations. (The fact that the total emission of the outer shells is significant is not in contradiction with the brightness drop with the radius in the images.)

The situation is significantly improved since, as we have seen, the photodissociation radius tends to be comparable to the distance at which underexcitation appears, at least for the two most often observed CO lines, $J=1-0$ and $J=2-1$. The contribution of the outer layers, beyond $R_{C O}$, can be neglected, just because there is no $\mathrm{CO}$ in them. This helps in the calculation of the $\mathrm{CO}$ emissivities. But, since the emission of the outer layers is important if $\mathrm{CO}$ is abundant in them, we find a strong dependence of the total calculated emission on the assumed value for $R_{C O}$ (this dependence is approximately linear when the emissivity is constant with the radius).

Finally we must mention that the ${ }^{12} \mathrm{CO}$ lines can be optically thick; but rarely they are very optically thick in all the points of the envelope and in all the directions, so, the 'growth curve' is never very flat and such an effect can be treated in the models. In this respect, observations of rare isotopes, mainly ${ }^{13} \mathrm{CO}$, help in the analysis.

Examples of the theoretical analysis of the CO line emission can be found in Justtanont et al. (1994), Groenewegen et al.(1998), Loup et al. (1993), and references therein.

\subsection{Observational results: mass loss rates}

The main expected result from the comparison of the $\mathrm{CO}$ observations and calculations is the determination of the mass loss rates $(\dot{M})$. For this task, we must know the $\mathrm{CO}$ relative abundance and photodissociation radius. The $\mathrm{CO}$ abundance is in fact determined in a selfconsistent way from models for the emission of several lines in well known sources: values between 3 and $810^{-4}$ are found. $R_{C O}$ is expected to depend on the mass loss rate, so both parameters must be calculated at the same time, by means of some kind of iterative procedure. In any case, the favorable $\mathrm{CO}$ excitation ensures that it is possible to calculate the 
mass loss rates from fitting of the observed intensities by means of models, or using relatively simple formulae (often obtained from parametrization of model grids). Comparison between different calculations indicate that the errors in the mass loss determinations are small, not exceeding a factor 3 . Those calculations, performed for hundreds of stars, constitute the most important element of knowledge on CSEs around AGB stars.

The mass loss rates determined by these means range between a few $10^{-8}$ and a few $10^{-5} M_{\odot} \mathrm{yr}^{-1}$, being in general well correlated with the FIR intensity and the dust mass loss rates determined from it (the gas to dust mass ratio ranges between 150 and 500).

For more details, see the very useful and thoughtful compilation by Loup et al. (1993), and the surveys by Knapp and Morris (1985; a pioneering, important work), Margulis et al. (1990; on CO in nearby stars), Olofsson et al. (1993a; on diffuse C-rich envelopes), Kerschbaum et al. (1996, 1998; on emission from semiregular and irregular variables), Young (1995; containing $J=3-2$ and $J=4-3$ data), Omont et al. (1993a; on very thick and cold envelopes), etc.

Both of the above limits are interesting. Except for a few objects (that are probably peculiar from the chemical point of view, see Kerschbaum et al. 1996, 1998), AGB stars show mass loss rates larger than $10^{-8} M_{\odot} \mathrm{yr}^{-1}$. It is of course difficult to work with these lower limits, because of the uncertain interpretation of the sample meaning and nondetections in the different papers. But it is remarkable that, even in surveys of diffuse envelopes, the detection rate is very high. Olofsson et al. (1993a) observed a 'relatively complete' sample of C-rich AGB stars up to a distance of $900 \mathrm{pc}$, up to $600 \mathrm{pc}$ all the objects were detected and show values of $\dot{M}$ over the limit given above. Other surveys, more or less biased towards dense envelopes, give similar results. No definitive conclusion can be drawn on this important subject, but it seems that at least an important subsample of the AGB stars (perhaps limited by the stellar mass) are loosing mass with $\dot{M}>10^{-8} M_{\odot} \mathrm{yr}^{-1}$.

The upper limit is also interesting. It is hard to find AGB stars with $\dot{M}$ higher than $10^{-5} M_{\odot} \mathrm{yr}^{-1}$. For instance, the largest value listed by Loup et al. (1993) for an AGB star is $410^{-5} M_{\odot} \mathrm{yr}^{-1}$ (but see Justtanont et al. 1994), and the famous, dense envelope of IRC +10216 has $\dot{M} \sim 210^{-5} M_{\odot} \mathrm{yr}^{-1}$ (Kastner 1992, Groenewegen et al. 1998). However, the mass loss rates measured in protoplanetary (post-AGB) objects, determined from the values of the total nebular mass $\left(\sim 0.1-1 \mathrm{M}_{\odot}\right)$ and the life time $(\sim 500-1000 \mathrm{yr})$, are larger than or about $10^{-4} M_{\odot} \mathrm{yr}^{-1}$ in most well studied cases (as for example in CRL 618, CRL 2343, CRL 2688, IRC +10420, NGC 7027, OH 231.8+4.2, Roberts 22, the Boomerang Nebula, etc). This means that the long-lasting mass loss process detected in AGB stars is not responsible for the ejection of the planetary nebula. Instead, we should invoke a discrete phenomenon: a copious, quasi-explosive ejection of matter, that also triggers the end of the AGB phase.

\subsection{Observational results: line profiles and envelope structure}

We will only marginally deal, for the sake of brevity, on the theoretical and observational characteristics of the molecular line profiles in AGB stars. It is well known (e.g. Morris 1975, Olofsson et al. 1982) that the line shape depends on the opacity and degree of spatial resolution attained by the used telescope. 
Two-peak profiles are observed in optically thin lines and (at least) partially resolved envelopes. Parabolic shapes appear in optically thick lines. A variety of intermediate cases are possible, with also some exceptions and deviations from the standard scheme (e.g. Bujarrabal et al. 1989, Knapp et al. 1998). The main parameters determined from the line profiles are the systemic and circumstellar expansion velocities, that correspond respectively to the line centroid and to half the observed line width; these parameters have been accurately measured in hundreds of stars (see data in Loup et al. 1993).

Our knowledge on the spatial structure of $\mathrm{CO}$ envelopes has recently received an important boost thanks to the publication of the IRAM atlas of CSEs (Neri et al. 1998). The AGB envelopes systematically show a circular CO image, again in opposition to the case of protoplanetary nebulae (that are mostly bipolar), as already shown by previous works (e.g. Stanek et al. 1995). In the accurate measurements by Neri et al., less than $20 \%$ of the $\mathrm{CO}$ images of AGB objects show a significant deviation from circular symmetry. In general, the measured radial decrease of the intensity is quite in agreement with the theoretical predictions. The measured outer radii range between $10^{16}$ and a few times $10^{17} \mathrm{~cm}$, increasing for objects with large mass loss rates. However, it has been impossible in practice to check the theoretical values of the photodissociation radius. There are good indications that the actual total $\mathrm{CO}$ extent is comparable to the theoretical $R_{C O}$ (Neri et al.), but the measured outer radii (for instance, the last significant contour in maps) remain somewhat smaller than the theoretical values. The reason is simply that the dynamic range of the existing interferometric maps is not high enough to reach the low brightness expected close to $R_{C O}$. (Single dish data seem to reach the required sensitivity in some published maps, but the poor spatial resolution and/or small map extent strongly hamper the outer radius measurement.) In any case, the present instrumental performances should allow observations accurate enough, and we can hope to get soon direct information on this important question.

The agreement between the radial variation of the $\mathrm{CO}$ brightness and the predictions by simple models suggests that in many objects, about $70 \%$ of the sample by Neri et al. (1998), the mass loss rate has been practically constant in the last few thousand years. But it is clear that there are sudden changes in the brightness in a nonnegligible number of objects, that probably correspond to changes in the ejection rate (see also Justtanont et al. 1996, Knapp et al. 1998). The case of the detached envelopes is particularly interesting. In these objects, a very thin molecular shell surrounds an AGB star, being clearly detached from it. In general there is also a more compact component very close to the star, characterized by lower mass loss rate and expansion velocity. Probably the $\mathrm{CO}$ image of the detached shell is limited by the ejection duration, though that of the compact component is limited by photodissociation. There is an interesting poster in this Symposium on this topic (Olofsson et al.), see also the published work by Olofsson and coworkers (1998a, and references therein).

\section{Other molecules}

The main purpose of the observation of the thermal emission from other molecules, less abundant than $\mathrm{CO}$, is the determination of their fractional abundance, the main empirical input to understand the very rich circumstellar chemistry. 
The theoretical understanding of the emission from these molecules is much worse than for $\mathrm{CO}$. There are two reasons for that : a) The excitation of these molecules is often more difficult to model than for $\mathrm{CO}$, because in most cases the level structures are more complex and the emission takes place under a regime of 'underexcitation' (as defined above). b) It is impossible that the big effort devoted to understand the $\mathrm{CO}$ line excitation can be also devoted to each of the many molecules found in AGB envelopes. So, in most cases only very simple calculations of the molecular abundances are performed, often assuming optically thin emission (not justified for some molecules, like $\mathrm{SiO}$ and $\mathrm{HCN}$ ), an outer radius (usually of the order of $10^{16} \mathrm{~cm}$ ) and a typical rotational temperature (derived from the observation of several transitions of the molecule). Under these conditions, the results only have a statistical meaning and differences as high as a factor ten can appear between different works.

Only in the case of the very well studied source IRC +10216 and/or for a few interesting molecules, the abundance determination is more sophisticated. In these cases, the studies are of course helped by a large observational information, including maps with high spatial resolution and multiline measurements. These well studied cases are discussed in the reviews by Lucas \& Guélin and Glassgold in this volume. A. Glassgold has also explained the main features of the chemistry models. For detailed analysis of molecule abundances, see the papers by Omont et al. (1993b) and Sahai \& Wannier (1992) on S-bearing molecules, by Bujarrabal et al. (1989) and Lucas et al. (1992) on SiO, by Nercessian et al. (1989), Fuente et al. (1998) and Olofsson et al. (1993b) on HCN and CN, etc. For the search of more exotic molecules, see for instance Cernicharo \& Guélin (1987) and Guélin et al. (1995). Isotopic abundance ratios have been studied in depth by Kahane et al. (1992).

Probably the most complete set of molecular abundances in AGB stars is still that calculated by Nyman et al. (1993b) for two C-rich stars, IRC +10216 and IRAS 15194-5115. (IRAS 15194-5115 is a southern star with a mass loss rate comparable to that of IRC +10216 , but placed much farther, at a distance of about $1200 \mathrm{pc}$.) These authors derived abundances in both sources for 18 species, including some isotopic substitutions. Even relatively heavy molecules, like $\mathrm{HC}_{3} \mathrm{~N}, \mathrm{C}_{3} \mathrm{~N}$ and $\mathrm{SiC}_{2}$, show high abundances, $>10^{-7}$. Both stars show a very similar chemistry, with abundances coincident within a factor 3 . We note that IRC +10216 and IRAS 15194-5115 are quite similar from other points of view, and that we lack for information on many of these species for objects with significantly different physical conditions and $\mathrm{O} / \mathrm{C}$ abundance.

\subsection{Dependence on the $\mathrm{O} / \mathrm{C}$ abundance ratio}

Due to the efficient formation of $\mathrm{CO}$, the $\mathrm{O} / \mathrm{C}$ relative abundance plays a very important role in the chemistry of the stellar atmosphere and the circumstellar envelope. In carbon rich objects, almost all $\mathrm{O}$ nuclei are locked in $\mathrm{CO}$; so, the O-bearing molecules are rare, while the remaining $\mathrm{C}$ serves to form the (many) possible carbon-bearing molecules. The opposite situation occurs in Orich envelopes. The first systematic, quantitative determination of the different molecular emission properties of $\mathrm{O}$ and $\mathrm{C}$-rich envelopes has been performed in Bujarrabal et al. (1994a,b), from observations of a sample of 47 AGB stars. As expected, the lines of $\mathrm{C}(\mathrm{O})$-bearing molecules are systematically much more in- 
tense in $\mathrm{C}(\mathrm{O})$-rich stars. For instance the $\mathrm{SiO}(J=2-1) / \mathrm{HCN}(J=1-0)$ line intensity ratio was found to be always larger than 2.3 in O-rich stars, while this ratio is smaller than 0.23 in all the studied carbon rich objects (note that these figures are obtained from main-beam temperatures and slightly depend on the telescope used). Similar quantitative conclusions were reached for other molecules, namely $\mathrm{SO}, \mathrm{HNC}, \mathrm{HC}_{3} \mathrm{~N}, \mathrm{SiS}$ (that behaves as the C-bearing molecules), and $\mathrm{CS}$. $\mathrm{CO}$ was also observed, its behavior being intermediate (as expected) between that of $\mathrm{C}$ - and O-bearing molecules. These results were recently confirmed by Olofsson et al. (1998b), for a larger sample of stars. For recent studies on S-stars, see Bieging et al. (1998).

The empirical discrimination is so clear that it can be applied to determine the $\mathrm{O} / \mathrm{C}$-rich nature of envelopes of uncertain classification by other means. It is remarkable that $\mathrm{S}$-type stars are intermediate in this respect between the $\mathrm{O}$ and C-rich groups, which shows that their envelopes are also S-type.

These works also allowed statistical studies of the molecule abundances for the different types of stars. The variations of the abundances (averaged for the detections) between $\mathrm{O}$ - and $\mathrm{C}$-rich stars are in general of about a factor ten (except for $\mathrm{CO}$ ). It is in particular noticeable that the abundance of $\mathrm{C}(\mathrm{O})$ bearing molecules in $\mathrm{O}(\mathrm{C})$-rich stars is in some cases not negligible. For instance, the average abundance of $\mathrm{HCN}$ in $\mathrm{O}$-rich envelopes was found to be $\sim 410^{-7}$, with a big dispersion, being its $J=1-0$ transition detected in the majority of the O-rich objects observed. On the other hand, $\mathrm{HC}_{3} \mathrm{~N}$ was not detected in the $8 \mathrm{O}$ rich stars observed in its $J=10-9$ transition, with upper limits to the abundance $\sim 10^{-7}, 20$ times smaller than the average abundance in C-rich stars.

\section{Maser transitions}

Maser effect appears in a transition when the populations of the joined levels are 'inverted', or, equivalently, when the excitation temperature is negative and the (also negative) absorption coefficient becomes an amplification coefficient. For large absolute values of the opacity, the exponential amplification yields very intense lines and strong contrasts in the spatial structure and line shape. For these reasons, masers are easily observed. There are data on about 2000 stars, including VLBI experiments with spatial resolution as high as 1 mas.

The key to understand maser emission is the pumping: the mechanism that maintains the population inversion. Very fine work has been published on the relevant microprocesses, that are interesting by themselves, but the pumping is often so complex that it is very difficult to derive physical or chemical conditions from comparison between models and observations. In fact, the maser observations are more efficient to determine the spatial and kinetic structure of the emitting regions. $\mathrm{OH}$ masers have been also useful to study the AGB stellar populations, including the detection and study of the $\mathrm{OH} / \mathrm{IR}$ stars (very red, optically unidentified AGB stars).

Several molecules show maser emission in AGB CSEs, the most intense masers being those of $\mathrm{OH}, \mathrm{H}_{2} \mathrm{O}$, and $\mathrm{SiO}$. The different lines come from different regions of the envelope, so they can be used to study the various circumstellar components. $\mathrm{SiO} \mathrm{mm}$-wave masers arise in the very inner shells (at a few $10^{14} \mathrm{~cm}$ from the star), $\mathrm{OH} 18 \mathrm{~cm}$ emission comes from much outer regions $\left(\gtrsim 10^{16} \mathrm{~cm}\right)$ 
and the $\mathrm{H}_{2} \mathrm{O} 1.3 \mathrm{~cm}$ line comes from intermediate layers $\left(2-1010^{14} \mathrm{~cm}\right)$. We can only discuss in this paper the case of $\mathrm{SiO}$ masers; for a quite complete review on circumstellar masers, and in general on AGB CSEs, see Habing (1996).

\subsection{SiO masers}

$\mathrm{SiO}$ shows maser emission in rotational transitions within excited vibrational states. Transitions from $J=1-0$ up to $J=8-7$ in the states $v=1,2,3,4$ have been observed, the total star sample including more than 500 stars. The $J=1-0$ transitions of $v=1,2$ and the $J=2-1$ transition of $v=1$ show the most intense masers. The observed line profiles are often composed of several spikes spread over a few $\mathrm{km} \mathrm{s}^{-1}$, and are centered on the stellar velocity. Weaker masers in ${ }^{29} \mathrm{SiO}$ and ${ }^{30} \mathrm{SiO}$ are also found, in this case also in the $v=0$ state. See for surveys of $\mathrm{SiO}$ masers, including observations of different lines: Jewell et al. (1987), Bujarrabal et al. (1987), Cernicharo et al. (1992, 1993), Nyman et al. (1993a), Izumiura et al. (1995), and references therein.

$\mathrm{SiO}$ masers are mostly detected in Mira-type variables, but they are also found in semiregular and irregular giants and some red supergiants (as well as in a few starforming regions and protoplanetary nebulae); $\mathrm{SiO}$ emitters are always O-rich, except for a few S-type stars. Miras show a very good correlation between $\mathrm{SiO}$ and IR absolute fluxes (Bujarrabal et al. 1987). The correlation is particularly good for the IR flux at $8 \mu \mathrm{m}$, which is the wavelength of the $\Delta v=1$ vibrational transition; this transition is thought to be responsible for the maser pumping in the radiative mechanisms (see below). Semiregular and irregular variables (including supergiants) are often weaker, i.e. they show a lower $\mathrm{SiO} / \mathrm{IR}$ flux ratio. Only objects of these types showing a variability amplitude in the visible larger than $2.5 \mathrm{mag}$ are comparable to Miras in $\mathrm{SiO}$ intensity, see Alcolea et al. (1990), Hall et al. (1990). (Remember that Miras are regular variable stars satisfying this requirement.)

$\mathrm{SiO}$ masers are found to be linearly polarized, with a degree that can reach almost $100 \%$ in some spikes, and in the average is $\sim 30 \%$; see e.g. Clark et al. (1985). Circular polarization is also detected, but not exceeding $\sim 10 \%$.

VLBI data show that $\mathrm{SiO}$ masers are placed in a ring-like structure surrounding the star at a distance of few stellar radii, see details in the contribution by Diamond \& Kemball in this volume. Other VLBI observations have also yielded interesting results, like the very small size of the emitting spots $\left(\sim 10^{12} \mathrm{~cm}\right.$, Colomer et al. 1992) and the partial coincidence between the clumps found in the $J=1-0 v=1$ and $v=2$ transitions (Miyoshi et al. 1994).

Finally, monitoring observations have shown that the $\mathrm{SiO}$ masers vary with the same period as the central star. There is a remarkable coincidence in phase between the variability curves of the $\mathrm{SiO}$ maser emission and of the IR; but this is not the case of the optical curve, since the $\mathrm{SiO}$ and IR maxima appear at phase $0.1-0.2$ with respect to the optical maxima. See the Yebes monitoring, the most complete one performed to date, in Alcolea et al. (1999).

The basic mechanism in the inversion of ${ }^{28} \mathrm{SiO}$ masers was discovered by Kwan \& Scoville (1974). In this process, the rotational lines for $v>0$ are inverted due to photon trapping in the $v \rightarrow v-1$ radiative deexcitation. The modified transition probability favors the vibrational components from the lowest $J$-levels, and so systematically overpopulates levels with high $J$ in $v$. What 
is still under discussion is the way in which the $v>0$ states are significantly populated in AGB envelopes. There are two basic pumping mechanisms, collisional (Lockett \& Elitzur 1992; Doel et al. 1995) and radiative (Bujarrabal 1994, and references therein).

It has been shown that collisions up to an excited vibrational state do not show any significant selection of the rotational levels, so the subsequent radiative decay can yield the observed population inversion. From the theoretical point of view, the advantage of the collisional pumping is that it only requires the existence, close to the star, of temperatures $\gtrsim 1500 \mathrm{~K}$ and a high $\mathrm{SiO}$ abundance; although, on the other hand, its efficiency is low. The collisional mechanism has problems to explain some observational results, like the relations between the $\mathrm{SiO}$ and IR intensities shown above.

In the radiative pumping, molecules are vibrationally excited by means of the absorption of stellar photons at $8 \mu \mathrm{m}$. The mechanism needs that the high opacity applied to the decay does not hold for the stellar photons traversing the maser region, which occurs in regions with high velocity gradients or shell-like shape. This requirement is the main drawback of the radiative pumping, though it is compatible with the observations. The radiative pumping naturally explains the correlation between the maser and IR intensities and the linear polarization, that in this case would appear not as a consequence of a magnetic field, but just as due to the anisotropic excitation. On the other hand, the coincidence between spots of different lines (see above) is more convincingly explained by the collisional pumping models.

Acknowledgments. This work has been supported by the Spanish DGES, under project PB96-0104, and by a grant from the IAU Symposium n ${ }^{\circ} 191$ Local Organizing Committee. I am also grateful to J. Alcolea and T. Le Bertre for critical reading of the manuscript.

\section{References}

Alcolea J., Bujarrabal V., Gómez-González J., 1990, A\&A 231, 431

Alcolea J., Pardo J.R., Bujarrabal V., et al., 1999, A\&AS, submitted

Bieging J.H., Knee L.B.G., Latter W.B., Olofsson H., 1998, A\&A 339, 811

Bujarrabal V., 1994, A\&A 285, 953

Bujarrabal V., Fuente A., Omont A., 1994a, A\&A 285, 247

Bujarrabal V., Fuente A., Omont A., 1994b, ApJ 421, L47

Bujarrabal V., Planesas P., Del Romero A., 1987, A\&A 175, 164

Bujarrabal V., Gómez-González J., Planesas P., 1989, A\&A 219, 256

Cernicharo J., Bujarrabal V., 1992, ApJ 401, L109

Cernicharo J., Bujarrabal V., Santarén J.L., 1993, ApJ 407, L33

Cernicharo J., Guélin M., 1987, A\&A 183, L10

Clark F.O, Troland T.H., Miller J.S., 1985, ApJ 289, 756

Colomer F., Graham D.A., Krichbaum T.P., et al., 1992, A\&A 254, L17

Doel R.C., Gray M.D., Humphreys E.M.L., et al., 1995, A\&A 302, 797

Fuente A., Cernicharo J., Omont A., 1998, A\&A 330, 232 
Goldreich P., Scoville N., 1976, ApJ 205, 144

Groenewegen M.A.T., 1994, A\&A 290, 531

Groenewegen M.A.T., van der Veen W.E.C.J., Matthews H.E., 1998, A\&A 338, 491

Guélin M., Forestini M., Valiron P., et al., 1995, A\&A 297, 183

Habing H.J., 1996, A\&A Rev 7, 97

Hall P.J., Allen D.A., Troup D.R., et al., 1990, MNRAS 243, 480

Izumiura H., Deguchi T., Hashimoto O, et al., 1995, ApJ 453, 837

Jewell P.R., Dickinson D.F., Snyder L.E., Clemens D.P., 1987, ApJ 323, 749

Justtanont K., Skinner C.J., Tielens A.G.G.M., 1994, ApJ 435, 852

Justtanont K., Skinner C.J., Tielens A.G.G.M., et al., 1996, ApJ 456, 337

Kahane C., Cernicharo J., Gómez-González J., Guélin M., 1992, A\&A 256, 235

Kastner J.H., 1992, ApJ 401, 337

Kerschbaum F., Olofsson H., Hron J., 1996, A\&A 311, 273

Kerschbaum F., Olofsson H., 1998, A\&A 336, 654

Knapp G.R., Morris M., 1985, ApJ 292, 640

Knapp G.R., Young K., Lee E., Jorissen A., 1998, ApJS 117, 209

Kwan J., Linke R.A., 1982, ApJ 254, 587

Kwan J., Scoville N., 1974, ApJ 194, L97

Lafont S., Lucas R., Omont A., 1982, A\&A 106, 201

Lockett P., Elitzur M., 1992, ApJ 399, 704

Loup C., Forveille T., Omont A., Paul J.F., 1993, A\&AS 99, 291

Lucas R., Bujarrabal V., Guilloteau S., et al., 1992, A\&A 262, 491

Mamon G.A., Glassgold A.E., Huggins P.J., 1988, ApJ 328, 797

Margulis M., Van Blerkom D.J., Snell R.L., Kleinmann S.G., 1990, ApJ 361, 673

Miyoshi M., Matsumoto K., Kameno S., et al., 1994, Nature 371, 395

Morris M., 1975, ApJ 197, 603

Nercessian E. Guilloteau S., Omont A., Benayoun J.J., 1989, A\&A 210, 225

Neri R., Kahane C., Lucas R., Bujarrabal V., Loup C., 1998, A\&AS 130, 1

Nyman L.-A., Hall P.J., Le Bertre T., 1993a, A\&A 280, 551

Nyman L.- $\AA$., Olofsson H., Johansson L.E.B., et al., 1993b, A\&A 269, 377

Olofsson H., Bergman P., Lucas R., et al., 1998a, A\&A 330, L1

Olofsson H., Eriksson K., Gustafsson B., Carlström U., 1993a, ApJS 87, 267

Olofsson H., Eriksson K., Gustafsson B., Carlström U., 1993b, ApJS 87, 305

Olofsson H., Johansson L.E.B., Hjalmarson $\AA$, Nguyen-Q-Rieu, 1982, A\&A 107, 128

Olofsson H., Lindqvist M., Nyman L.- $\AA$., Winnberg A., 1998b, A\&A 329, 1059

Omont A., Loup C., Forveille T., et al., 1993a, A\&A 267, 515

Omont A., Lucas R., Morris M., Guilloteau S., 1993b, A\&A 267, 490

Sahai R., Wannier P.G., 1992, ApJ 394, 320

Stanek K.Z., Knapp G.R., Young K., Phillips T.G., 1995, ApJS 100, 169

Young K., 1995, ApJ 445, 872 